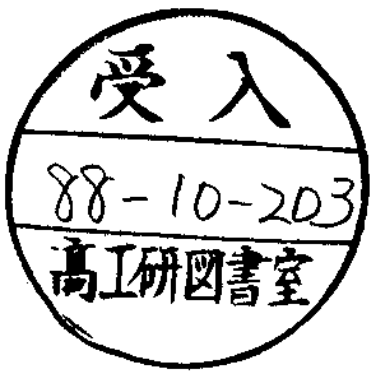

CERN-TH-5175/88

September 1988

\title{
Weak Matrix Elements at Strong Coupling
}

\author{
Apoorva Patel
}

Theory Division, CERN, Geneva, Switzerland

Talk presented at the Ringberg Workshop on Hadronic Matrix Elements and Weak Decays Ringberg Castle, Federal Republic of Germany, April 18-22, 1988

\begin{abstract}
I summarise the results for hadronic matrix elements appearing in non-leptonic weak amplitudes calculated in the strong coupling limit of lattice QCD.
\end{abstract}

CERN-TH-5175/88

September 1988 
Most of what I am going to report here has already been presented at Seillac ${ }^{1}$. So I shall be brief, stressing only the key features.

In the effective field theory framework, the computation of amplitudes for electro-weak processes involves three steps ${ }^{2}$ : (a) Start with the standard $S U(3)_{c} \times S U(2)_{L} \times U(1)_{Y}$ model, and carry out a perturbative expansion in the electro-weak interactions. (b) Gradually lower the energy scale evolving the various interactions using the renormalisation group equations. (c) When the energy scale becomes comparable to a heavy field mass, integrate the heavy field out using the operator product expansion, leaving behind a Lagrangian containing effective interactions of the light fields. Repeating steps (b) and (c), one finally arrives at a Lagrangian containing renormalised interactions, whose matrix elements are to be evaluated at low energy scales :

$$
L_{\text {int }}=\sum_{i} C_{i}(\mu) O_{i}(\mu)
$$

Here both the effective operators $O_{i}$ and the Wilson coefficients $C_{i}$ are defined using a particular regularisation scheme and they depend on the measurement scale $\mu$. The matrix elements of $L_{\text {int }}$ between hadronic states however have no dependence either on the regularisation scheme or the scale $\mu$.

The coefficients $C_{i}$ are generally calculated using weak coupling perturbation theory, while the quantities $<O_{i}>\equiv<h_{f}\left|O_{i}\right| h_{i}>$ are estimated by non-perturbative methods. It is this latter part which requires the knowledge of how QCD contributions modify the amplitudes at low energy scales, particularly in the case of non-leptonic processes. The processes of interest at this conference are the ones dealing with the pseudo-Goldstone boson fields. In such cases, the pattern of chiral symmetry breaking restricts the form of the various amplitudes, and one hopes to achieve an understanding of the remaining parameters in a theoretical framework.

The lattice formulation of QCD provides an explicitly non-perturbative framework for evaluating the $\left\langle O_{i}\right\rangle$. In this regularisation, one can think of lowering the scale $\mu$ all the way down to the strong coupling limit, which has both confinement and spontaneous chiral symmetry breaking naturally built in. I present results for the $\left\langle O_{i}\right\rangle$ in this limit using the staggered fermion formulation. I prefer staggered fermions because they produce Goldstone bosons with the correct chiral behaviour by preserving a subset of the continuum chiral symmetries at all scales.

Before going on to the results, I would like to discuss the usefulness and limitations of such an approach :

- One is working at the "wrong" fixed point of the theory, and the weak coupling scaling behaviour is lost. Nonetheless, one still has a regulated theory of (partially) conserved currents and their corresponding charges, allowing explicit comparison of the results with the chiral Lagrangian description of pseudo-Goldstone boson interactions.

- The hadron spectrum cannot be fit to better than $\sim 30 \%$ accuracy. However, the results include contributions due to higher dimension operators and excited states without arbitrary parameters. The counter-terms of chiral perturbation theory are indirectly present in the regulated lattice theory, constraining its predictions.

- The complete set of continuum chiral symmetries does not exist on the lattice and mixing between different lattice operators takes place. On the other hand, the chiral 
behaviour of the amplitudes is not assumed but is a consequence. No restriction to small quark masses is necessary, and corrections to the leading chiral behaviour can be readily calculated.

- Perturbative calculations of $C_{i}$ and operator mixing cannot be trusted. They can only be regarded as a qualitative guide. But one can use continuous momenta and directly compute on-shell matrix elements.

- Though the final word on scaling tests and on removal of lattice artifacts is left to Monte Carlo calculations, the analytic results devoid of chiral extrapolations as well as of statistical and finite volume errors can provide useful guidelines.

These reasons make it clear that the results obtained in the strong coupling limit should be interpreted only as trends and not as quantitative predictions.

The strong coupling expansion is an expansion in powers of $1 / \mathrm{Ng}^{2}$ about the point $g^{2}=\infty$. So it can be thought of as a rearranged $1 / N$ expansion. At $g^{2}=\infty$ the gauge field action is zero, and the correlation functions can be expressed as diagrams containing random walks of colour singlet hadrons. These diagrams can be classified according to the powers of $N$ in their contributions. I will limit myself to calculating the first two nontrivial terms in such a $1 / N$ series, though terms of arbitrarily high order in $1 / N$ contribute even at $g^{2}=\infty$. As expected, the leading terms in this procedure reproduce the results of the vacuum insertion approximation (VIA). But it is also not difficult to see that the terms which are dropped are suppressed by powers of the hopping parameter in addition to powers of $1 / N$. An immediate corollary is that the VIA becomes exact for large quark masses irrespective of the $N$ value. This point has not received sufficient attention in the literature. It is somewhat surprising, as shown by the Monte Carlo results ${ }^{3}$, that the VIA indeed works very well all the way down to $m \approx m_{s}$.

The 4-Fermi operators of the weak interactions have the structure of a product of two currents. The transcription of these operators on the lattice obeying the Fierz and Ward identities involves split-point currents spread over a $2^{4}$ hypercube ${ }^{4,5}$. Though such a process typically gives rise to many terms, discrete symmetries restrict the number of terms contributing to a given process. The lattice transcription of staggered fermion correlation functions having the correct continuum limit is not unique. In the strong coupling linit, I choose to work with correlation functions written in a 1-spinor loop Fierz arrangement and containing 1-link currents as well as the exact Goldstone bosons. This selection of terms is based on a desire to use only the (partially) conserved vector and axial vector lattice currents while keeping the chiral symmetries intact. I optimistically hope that the conserved nature of the currents keeps the renormalisation and operator mixing effects close to those in the continuum. Recent progress in the calculation of 1-loop staggered fermion operator renormalisation ${ }^{6,5}$ will have more to say about this assumption.

At strong coupling, a correlation function of quark bilinears can be expressed as a sum of mesonic Feynman diagrams. The 2 -point lattice meson propagator was calculated ${ }^{7}$ by resumming a hopping parameter expansion. For simplicity, I only use the large $-d$ limits of those results :

$$
\kappa=\frac{1}{m+\sqrt{2 d+m^{2}}}, 4 \sinh ^{2}\left(\frac{m_{K}}{2}\right)=\frac{1}{\kappa_{s}}-2 d, f_{K}=\frac{\sqrt{N}}{2}\left(\kappa+\kappa_{s}\right) .
$$

Here $\kappa$ is the effective quark hopping parameter and the expressions are evaluated at 
leading order in $N$. In this order the weak matrix elements are $O\left(N^{2}\right)$. They completely factorise and are identical to the VIA results. Up to the same order the Wilson coefficients $C_{i}(\mu)$ are independent of the scale $\mu$, and no QCD effects appear.

The QCD effects show up at the next order in $N$. New operators $O_{i}$ enter the picture, giving rise to correlation functions of $O(N)$. An anomalous dimension matrix appears as well, producing renormalisation group scaling of the Wilson coefficients $C_{i}(\mu)$. In addition to these there are $1 / N$ corrections (such as due to dynamical fermions) to the leading $O\left(N^{2}\right)$ correlation functions. These last kind of $1 / N$ effects can be completely absorbed in quantities like $m_{\pi}, f_{\pi}$ and normalisation constants, due to the factorisability of the $O\left(N^{2}\right)$ correlation functions. Therefore, though the physical value of the lattice spacing gets altered, dimensionless numbers can be consistently extracted using the expressions in eq.(2) in all the correlation functions.

A matrix element which does not suffer from uncertainties of renormalisation and operator mixing is the electromagnetic contribution to the pion mass difference. It was calculated to be $\mathrm{b}^{7}$, using $f_{\pi}$ to fix the lattice scale, $m_{\pi^{+}}^{2}-m_{\pi^{0}}^{2}=(41.3 \mathrm{MeV})^{2}$, compared to the experimental value of $(35.6 \mathrm{MeV})^{2}$. It is also simple to calculate the contribution of the box diagram $|\Delta S|=2$ operator to $K^{0}-\bar{K}^{0}$ mixing :

$$
\left\langle K^{0}\left|(\bar{d} s)_{V-A}(\bar{d} s)_{V-A}\right| \widehat{K}^{0}\right\rangle=2 f_{K}^{2} M_{K}^{2}\left(1+\frac{1}{N}\right), M_{K}=2 \sinh \left(\frac{m_{K}}{2}\right) .
$$

The $K \rightarrow 2 \pi$ decays require computations of 4 -point functions. The relevant 4 -Fermi $|\Delta S|=1$ operators (without integrating the charm quark out) are :

$$
O_{ \pm}=\frac{1}{2}\left[(\bar{u} s)_{V-A}(\vec{d} u)_{V-A} \pm(\bar{d} s)_{V-A}(\bar{u} u)_{V-A}-(\bar{c} s)_{V-A}(\bar{d} c)_{V-A} \mp(\bar{d} s)_{V-A}(\bar{c} c)_{V-A}\right]
$$

Omitting a common factor of $G_{F} \sin \theta_{c} \cos \theta_{c} / \sqrt{2}$ for brevity, the initial condition on the Wilson coefficients at the weak scale is $C_{+}=C_{-}=1$. The 1 -loop QCD renormalisation effects $^{8}$ suppress $C_{+}$and enhance $C_{-}$. Moreover,

$$
C_{+}+C_{-}=O(1), C_{+}-C_{-}=O\left(\frac{1}{N}\right), C_{-}=\left(C_{+}\right)^{(1+N) /(1-N)} .
$$

The $\Delta I=3 / 2$ decay, $K^{+} \rightarrow \pi^{+} \pi^{0}$, gets contribution from tree level meson diagrams only :

$$
\left\langle K^{+}\left|O_{+}\right| \pi^{+} \pi^{0}\right\rangle=\frac{f_{\pi}}{\sqrt{2}}\left(M_{K}^{2}-M_{\pi}^{2}\right)(1-\delta)\left(1+\frac{1}{N}\right), \quad \delta=\frac{M_{\pi}^{2} \kappa^{2} \kappa_{s}}{\kappa+\kappa_{s}} .
$$

The ensuing prediction $\left\langle K^{+}\left|L_{\text {int }}\right| \pi^{+} \pi^{0}\right\rangle=C_{+}(\mu) \times 0.027 \mathrm{GeV}^{3}$ has to be contrasted with the experimental result $0.010 \mathrm{GeV}^{3}$.

The decay amplitude for $K^{0} \rightarrow \pi^{+} \pi^{-}$contains diagrams with an internal meson loop as well. With the charm quark in the internal loop, and $k_{\mu}$ and $p_{1 \mu}$ respectively denoting 
the four momenta of the $K^{0}$ and $\pi^{+}$, this contribution is :

$$
\begin{gathered}
I(c)=f_{\pi} \int_{-\pi}^{\pi} \frac{d^{4} l}{(2 \pi)^{4}}\left[\frac{\kappa_{s} \sum_{\mu} \cos (l-k)_{\mu}-\kappa \sum_{\mu} \cos \left(l-p_{1}\right)_{\mu}}{\kappa^{2}\left(\kappa+\kappa_{s}\right) W X Y Z}\right. \\
\cdot\left\{\left(\kappa-\kappa_{s}\right)\left(16 \kappa_{c}^{3}+2 \frac{\kappa_{c}^{3}}{\kappa_{s}}-2 \kappa_{c}\right)+4 \kappa_{c}^{2}\left(\kappa_{c}^{2}-\kappa \kappa_{s}\right) \sum_{\mu} \cos l_{\mu}\right\} \\
\left.+\frac{2 \kappa_{c}}{\kappa\left(\kappa+\kappa_{s}\right)} \sum_{\mu}\left\{\frac{\kappa+\kappa_{s}}{Y Z} \cos \left(l-p_{1}-k\right)_{\mu}-\frac{\kappa}{Y} \cos \left(l-2 p_{1}\right)_{\mu}-\frac{\kappa_{s}}{Z} \cos (l-2 k)_{\mu}\right\}\right] \\
W=1+2 \kappa_{s} \kappa_{c} \sum_{\mu} \cos l_{\mu}, X=1-2 \kappa \kappa_{c} \sum_{\mu} \cos l_{\mu} \\
Y=1+2 \kappa \kappa_{c} \sum_{\mu} \cos \left(l-p_{1}\right)_{\mu}, Z=1-2 \kappa \kappa_{c} \sum_{\mu} \cos (l-k)_{\mu}
\end{gathered}
$$

An error in the expression given in ref. 1 has been corrected here ${ }^{\S}$. Evaluating the integral over the lattice Brillouin zone numerically, I obtain $I(u)-I(c) \approx 0.76 f_{\pi}\left(M_{K}^{2}-M_{\pi}^{2}\right)$.

The decay $K^{0} \rightarrow \pi^{0} \pi^{0}$ has the same diagrams appearing with different coefficients. Putting the full matrix elements together

$$
\begin{aligned}
&\left\langle K^{0}\left|L_{i n t}\right| \pi^{+} \pi^{-}\right\rangle=\frac{f_{\pi}}{2}\left(M_{K}^{2}-M_{\pi}^{2}\right) {\left[C_{-}(\mu)\left(1-\frac{1}{N}\right)\left(1-\delta+\frac{\kappa_{s}}{\kappa}(1+\delta)+0.76\right)\right.} \\
&+\left.C_{+}(\mu)\left(1+\frac{1}{N}\right)\left(1-\delta-\frac{\kappa_{s}}{\kappa}(1+\delta)-0.76\right)\right] \\
&\left\langle K^{0}\left|L_{i n t}\right| \pi^{0} \pi^{0}\right\rangle=\frac{f_{\pi}}{2 \sqrt{2}}\left(M_{K}^{2}-M_{\pi}^{2}\right)\left[C_{-}(\mu)\left(1-\frac{1}{N}\right)\left(1-\delta+\frac{\kappa_{s}}{\kappa}(1+\delta)+0.76\right)\right. \\
&\left.-C_{+}(\mu)\left(1+\frac{1}{N}\right)\left(1-\delta+\frac{\kappa_{s}}{\kappa}(1+\delta)+0.76\right)\right]
\end{aligned}
$$

These have to be compared with the experimental numbers $0.156 \mathrm{GeV}^{3}$ and $0.105 \mathrm{GeV}^{3}$ respectively.

The matrix elements in eqs. $(6,9)$ have the correct behaviour in the chiral limit. Also it can be seen that the $K^{+} \rightarrow \pi^{+} \pi^{0}$ and the $K^{0} \rightarrow \pi^{+} \pi^{-}$decays are $O\left(N^{2}\right)$, but the $K^{-0} \rightarrow \pi^{0} \pi^{0}$ decay is $O(N)$. At this stage there is no real clue as to the appropriate values of the coefficients $C_{ \pm}(\mu)$ at the lattice momentum cut-off scale $\mu=\pi / a$. Since the lattice diagrams for $K^{+}$and $K^{-0}$ decays are of the same order of magnitude, the $|\Delta I|=1 / 2$ enhancement must come from the Wilson coefficients. This is quite unlike the situation at larger values of $\mu$, where the Wilson coefficients are close to 1 and the enhancement is expected from diagrams containing an internal quark loop.

Working backwards, it can be seen that $C_{+}(\mu)=0.35$ and $C_{-}(\mu)=7.2$ bring the predicted decay rates close to the experimental ones. These values of $C_{ \pm}$satisfy the last relation in eq.(5) reasonably well. In the leading order perturbation theory, this relation follows from the ratio of anomalous dimensions and is $\mu-$ independent. It seems to remain

$\S$ I discovered this error after giving the talk at Ringberg. 
approximately correct at much lower energy scales beyond the range of validity of the leading order perturbation theory.

Finally, I take this opportunity to point out that the objection raised by Banks ${ }^{9}$ on the realisation of staggered fermion symmetries is in error. In the continuous time limit, the Lagrangian staggered fermions reduce to two decoupled species of Hamiltonian staggered fermions with mass terms of opposite sign ${ }^{10}$. Noting that the $\gamma$-matrices appearing in the fermion determinant are even dimensional and that the operator $\not D$ is anti-Hermitian, we have

$$
\begin{gathered}
\operatorname{det}(\not D-m)=\operatorname{det}(-\not D+m)=\operatorname{det}\left(\not D^{\dagger}+m\right)=[\operatorname{det}(\not D+m)]^{*}, \\
<\operatorname{det}(\not D-m) \operatorname{det}(\not D+m)>=\left\langle|\operatorname{det}(\not D+m)|^{2}>\geq\left\langle\operatorname{det}^{2}(\not D+m)>.\right.\right.
\end{gathered}
$$

The inequality sign is exactly opposite to what Banks has, and therefore his argument proves just the opposite of his claim. The effective potential chooses the asymmetric vacuum, with the spontaneously broken even-odd symmetry being identified as chiral. The conventional interpretation of staggered fermion symmetries hence remains valid.

I thank A. J. Buras and J.-M. Gerard for organising a wonderful conference.

\section{REFERENCES}

1) A. Patel, in "Field Theory on Lattice : LAT87", eds. A. Billoire et al., Nucl. Phys. B (Proc. Suppl.) 4 (1988) 510.

2) A pedagogical discussion can be found in : H. Georgi, Weak Interactions and Modern Particle Theory (Benjamin/Cummings, 1984).

3) S. Sharpe, in "Lattice Gauge Theory 86", eds. H. Satz et al. (Plenum Press, New York, 1987) 355;

S. Sharpe, R. Gupta, G. Guralnik, G. Kilcup and A. Patel, Phys. Lett. B192 (1987) 149.

4) S. Sharpe, A. Patel, R. Gupta, G. Guralnik and G. Kilcup, Nucl. Phys. B286 (1987) 253.

5) S. Sharpe, this volume;

R. Gupta, G. Kilcup, A. Patel and S. Sharpe, in preparation.

6) D. Daniel and S. Sheard, Nucl. Phys. B302 (1988) 471;

S. Sheard, Edinburgh Preprint 88/439 (1988).

7) O. Martin and A. Patel, Phys. Lett. B174 (1986) 94.

8) M. Gaillard and B. Lee, Phys. Rev. Lett. 33 (1974) 108;

G. Altarelli and L. Maiani, Pliys. Lett. 52B (1974) 351.

9) T. Banks, Nucl. Phys. B278 (1986) 141.

I am grateful to Stephen Sharpe for prodding me on this point.

10) A. Patel, Phys. Lett. 141B (1984) 249. 